\title{
Collision Energy Dependence of State-to-State Differential Cross Sections for Rotationally Inelastic Scattering of $\mathrm{H}_{2} \mathrm{O}$ by $\mathrm{He}$
}

\author{
Gautam Sarma ${ }^{1}$, Ashim Kumar Saha ${ }^{1}$, Chandan Kumar Bishwakarma ${ }^{1}$, Roy Scheidsbach ${ }^{1}$, Chung-Hsin Yang ${ }^{1,2}$,
} David Parker ${ }^{1}$, Laurent Wiesenfeld ${ }^{3}$, Udo Buck ${ }^{4}$, Lazaros Mavridis ${ }^{5}$, Sarantos Marinakis ${ }^{5 *}$

1. Radboud University Nijmegen, Institute for Molecules and Materials, Nijmegen, the Netherlands

2. Institute of Atomic and Molecular Sciences, Academia Sinica, Taipei, Taiwan

3. Univ. Grenoble Alpes and CNRS, IPAG, F-38000 Grenoble, France

4. Max-Planck-Institut für Dynamik und Selbst-Organisation, Am Faßberg 17, D-37077 Göttingen, Germany

5. Department of Chemistry and Biochemistry, School of Biological and Chemical Sciences, Queen Mary University of London, Joseph Priestley Building, Mile End Road, London E1 4NS, UK

* corresponding author

\begin{abstract}
The inelastic scattering of $\mathrm{H}_{2} \mathrm{O}$ by $\mathrm{He}$ as a function of collision energy in the range $381 \mathrm{~cm}^{-1}$ to $763 \mathrm{~cm}^{-1}$ at an energy interval of approximately $100 \mathrm{~cm}^{-1}$ has been investigated in a crossed beam experiment using velocity map imaging. Change in collision energy was achieved by varying the collision angle between the $\mathrm{H}_{2} \mathrm{O}$ and $\mathrm{He}$ beam. We measured the state-to-state differential cross section (DCS) of scattered $\mathrm{H}_{2} \mathrm{O}$ products for the final rotational states $J_{K a K c}=1_{10}, 1_{11}, 2_{21}$ and $4_{14}$. Rotational excitation of $\mathrm{H}_{2} \mathrm{O}$ is probed by $(2+1)$ resonance enhanced multiphoton ionization (REMPI) spectroscopy. DCS measurements over a wide range of collision energles allowed us to probe the $\mathrm{H}_{2} \mathrm{O}$-He potential energy surface (PES) with greater detail than in previous work. We found that a classical approximation of rotational rainbows can predict the collision energy dependence of the DCS. Close-coupling quantum mechanical calculations were used to produce DCS and partial cross sections. The forward-backward ratio (FBR), is introduced here to compare the experimental and theoretical DCS. Both theory and experiments suggest that an increase in the collision energy is accompanied with more forward scattering.
\end{abstract}




\section{Introduction}

Water, the essential ingredient to the very existence of life, is prevalent in space and plays a vital role in star formation.[1] Emission due to rotational transitions permits its identification in dense interstellar clouds.[2] Observation of water in interstellar conditions has been one of the main goals of the space telescope Herschel.[3] The rotational population of $\mathrm{H}_{2} \mathrm{O}$ in molecular clouds is a trade-off between photonic and molecular collisiosn, mainly with $\mathrm{H}_{2}$ and $\mathrm{He}$, which are highly abundant in those environments. Therefore, the reliable modeling of $\mathrm{H}_{2} \mathrm{O}$ in interstellar space depends on the accuracy of its potential energy surface (PES) for interaction with $\mathrm{H}_{2}$ or $\mathrm{He}$.

The quest to understand the collisional excitation of interstellar $\mathrm{H}_{2} \mathrm{O}[4]$ has inspired several studies on the collision of $\mathrm{H}_{2} \mathrm{O}$ with He.[5-10] Potential energy parameters have been estimated for elastic scattering of $\mathrm{H}_{2} \mathrm{O}$ with $\mathrm{He}$ and several scattering partners in a molecular beam experiment.[5] Another similar experiment examined the van der Waals potential for interactions of $\mathrm{H}_{2} \mathrm{O}$ with $\mathrm{He}$ and revealed it to be less anisotropic than expected in both the attractive and repulsive part of the potential.[6] There have been previous crossed beam studies to probe the $\mathrm{Ar}-\mathrm{H}_{2} \mathrm{O}$ potential energy surface,[11] which might resemble the $\mathrm{He}-\mathrm{H}_{2} \mathrm{O}$ surface. However, due to the small radius and less polarizable nature of $\mathrm{He}$, the $\mathrm{He}-\mathrm{H}_{2} \mathrm{O}$ surface is a better probe of the molecular force fields of $\mathrm{H}_{2} \mathrm{O}$, particularly the repulsive part of the force fields.

State-of-the-art inelastic scattering studies of $\mathrm{H}_{2} \mathrm{O}$ with He have been performed recently[12, 13] with initial and final $\mathrm{H}_{2} \mathrm{O}$ state-to-state excitation and detection. This type of experiments provided state-to-state differential cross sections (DCSs), which can offer important information regarding the underlying PES. In those DCSs, a significant change towards backward scattering with increased $\mathrm{H}_{2} \mathrm{O}$ final rotational level was observed, suggesting the importance of hard collisions through the anisotropic repulsive part of the PES. Here, an extension of that study to various collision energies is presented, allowing the probe of a larger range of the PES. 


\section{Rotational energy levels of $\mathrm{H}_{2} \mathrm{O}$}

The rotational energy levels of $\mathrm{H}_{2} \mathrm{O}$ in its electronic and vibrational ground state are shown in Fig. 1 . The rotational levels of $\mathrm{H}_{2} \mathrm{O}$ are denoted by $J_{K a K c}$ notation, where $J$ is the total angular momentum of the molecule, and $K_{a}$ and $K_{c}$ the quantum numbers for the projection of $J$ on the $A$ and $C$ principal rotation axes of $\mathrm{H}_{2} \mathrm{O}$, respectively. As there are two identical $\mathrm{H}$ atoms in $\mathrm{H}_{2} \mathrm{O}$, it has two nuclear spin states: total nuclear spin $l=1$, orthc in this study.[11] The excited states of $\mathrm{H}_{2} \mathrm{O}$ probed in this study are indicated by dashed lines in Fig. 1.

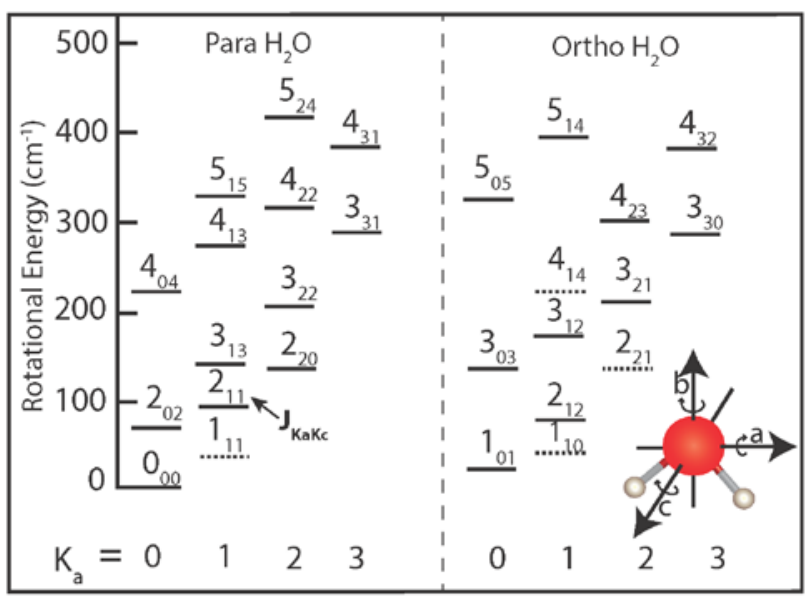

Figure 1: The first rotational energy levels of $\mathrm{H}_{2} \mathrm{O}$ in its ground electronic and vibrational state up to $500 \mathrm{~cm}^{-1}$. Levels are labelled $J_{K a K c}$ and are plotted versus the $K_{a}$ quantum number. The excited rotational levels probed in this work are depicted in dashed lines. 


\section{Methods}

\section{A. Experimental}

A schematic diagram of the crossed beam experimental setup is shown in Fig. 2. The experiments employed two differentially pumped beam sources, and a velocity map imaging (VMI) setup inside a vacuum chamber. A commercially available Jordan pulsed valve was used for the primary $\left(\mathrm{H}_{2} \mathrm{O}\right)$, and a General valve was used for the secondary $(\mathrm{He})$ beam. Both valves had a nozzle diameter of $0.5 \mathrm{~mm}$ and were operated at $10 \mathrm{~Hz}$. The pulse duration of the Jordan valve was around $100 \mu \mathrm{s}$, while the pulse duration of the General valve was around $300 \mu \mathrm{s}$. The molecular beam of $\mathrm{H}_{2} \mathrm{O}$ was produced by supersonic expansion of a $\mathrm{H}_{2} \mathrm{O}-\mathrm{Ar}$ mixturecreated by flowing 1 bar of pure $\mathrm{Ar}$ gas into a $\mathrm{H}_{2} \mathrm{O}$ bubbler containing demineralized water at room temperature. The concentration of $\mathrm{H}_{2} \mathrm{O}$ in the primary beam was $2.5 \%$ as determined by the vapor pressure of $\mathrm{H}_{2} \mathrm{O}$ at room temperature and 1 bar backing pressure of $\mathrm{Ar}$.

Due to adiabatic expansion, $\mathrm{H}_{2} \mathrm{O}$ in the beam was rotationally cooled to $\sim 12 \mathrm{~K}$. At that rotational temperature, $90 \%$ of ortho- $\mathrm{H}_{2} \mathrm{O}$ was in its lowest $1_{01}$ level, and $97 \%$ of para- $\mathrm{H}_{2} \mathrm{O}$ was in its lowest $\mathrm{O}_{00}$ level. The primary beam contained $\sim 10 \%$ of ortho- $\mathrm{H}_{2} \mathrm{O}$ in the $1_{10}$ level, and $\sim 3 \%$ of para- $\mathrm{H}_{2} \mathrm{O}$ in the $1_{11}$ level. Both the primary and secondary beams were collimated by a skimmer of $2.5 \mathrm{~mm}$ diameter positioned $30 \mathrm{~mm}$ downstream from the valve nozzle. The angle between the two molecular beams could be varied by rotating the primary beam around the center of the ion optics in the plane of collision. The primary beam could be positioned at any angle from 60 to $180^{\circ}$ with respect to the secondary beam in order to allow measurements to be carried out in a wide range of collision energies under the same experimental conditions. By varying the angle between the two molecular beams, the collision energy $\left(E_{\mathrm{col}}\right)$ in the center-of-mass frame is given by the following equation

$$
E_{\mathrm{col}}=\frac{1}{2} \mu v_{\mathrm{rel}}^{2}
$$

and 


$$
v_{\text {rel }}^{2}=v_{1}^{2}+v_{2}^{2}-2 v_{1} v_{2} \cos \theta
$$

where $v_{1}$ and $v_{2}$ are the velocities of the two beams in the laboratory frame, and $\theta$ is the crossing angle of the two beams. 


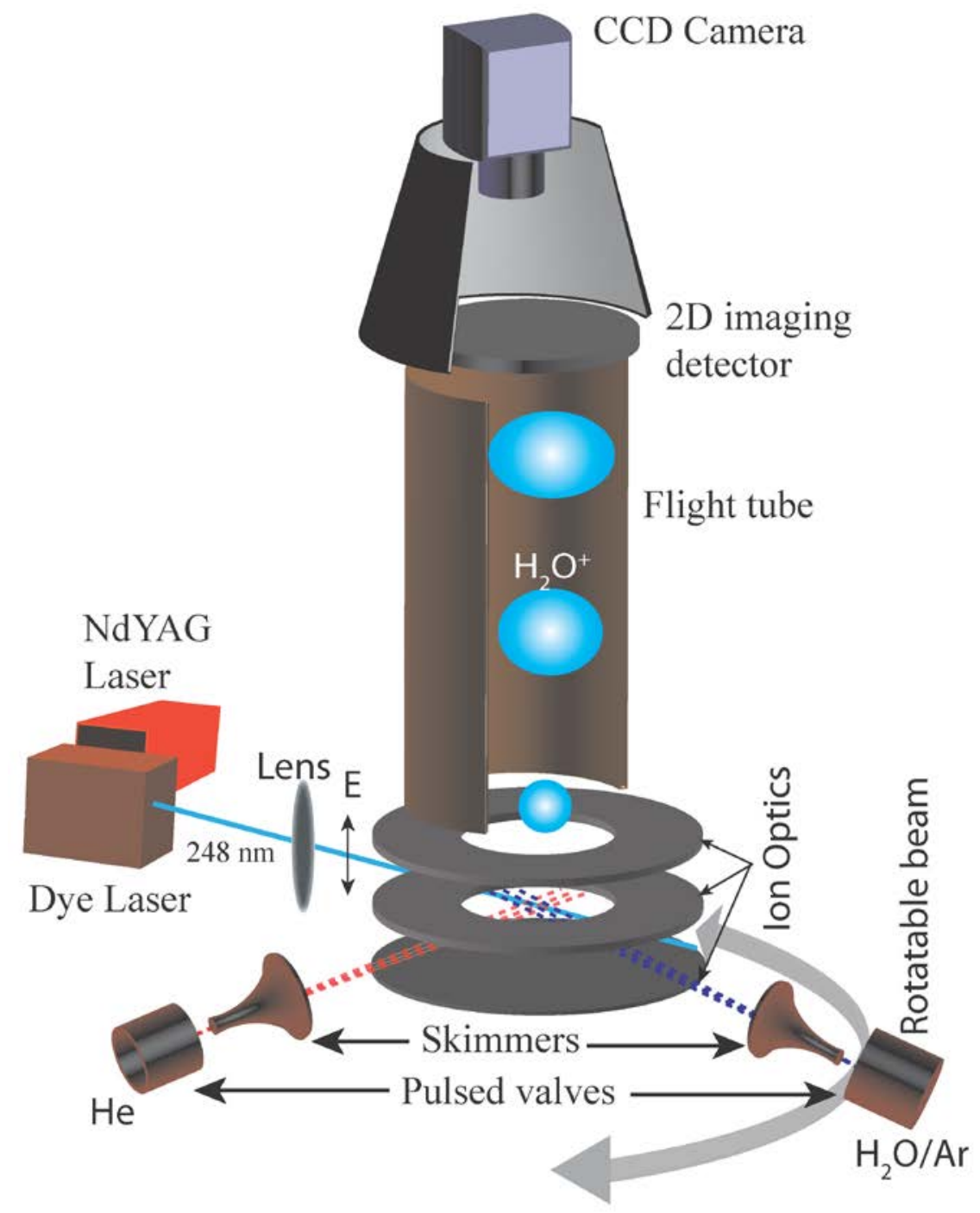

Figure 2: Schematic diagram of the crossed-beam VMI experimental setup. Pulsed molecular beam expansions of pure $\mathrm{He}$, and $\mathrm{H}_{2} \mathrm{O}$ in $\mathrm{Ar}$ are skimmed and crossed at various angles. Rotationally excited $\mathrm{H}_{2} \mathrm{O}$ are state-selectively ionized using a focused $(20 \mathrm{~cm}$ lens) pulsed tunable laser beam around $248 \mathrm{~nm}$ and $(2+1)$ REMPI via the C-state. $\quad \mathrm{H}_{2} \mathrm{O}^{+}$ions are mass-selected by time-of-flight, and then projected by a VMI lens onto a two-dimensional imaging detector monitored by a charge coupled device (CCD) camera. 
$\mathrm{H}_{2} \mathrm{O}$ molecules were rotational state selectively ionized by $(2+1)$ resonance enhanced multiphoton ionization (REMPI) spectroscopy using $\sim 248 \mathrm{~nm}$ laser radiation. The ionization radiation was generated using a dye laser system (Lambda Physik ScanMate) pumped by the third harmonic of a pulsed Nd:YAG laser (Continuum Powerlite 9010). Coumarin 307 dye was used in the dye laser to produce tunable laser light at a a wavelength around $496 \mathrm{~nm}$. This output was then frequency doubled using a barium borate (BBO) crystal to produce the required ionization photons at wavelength $\sim 248 \mathrm{~nm}$. The laser was operated at a repetition rate of $10 \mathrm{~Hz}$, with pulse energies of 3-4 $\mathrm{mJ}$. A spherical lens of $50 \mathrm{~cm}$ focal length was used to focus the ionization laser to the center of the collision region and the ion optics.

$\mathrm{H}_{2} \mathrm{O}^{+}$ions were projected to the detector due to electric potentials applied to a set of electrode plates (ion optics) called repeller and extractor. The ion optics were positioned in the center of the collision chamber and mounted in such a way that the time-of-flight axis is perpendicular to the collision plane. The sphere of $\mathrm{H}_{2} \mathrm{O}^{+}$ions was crushed onto a position sensitive micro-channel plates (MCP) detector. Mass selectivity in the detection was obtained by switching on the MCP by a pulse voltage at the appropriate moment. The size of the ion sphere on the detector is inversely proportional to the square root of the repeller voltage. We applied a rather low repeller plate voltage $(1000 \mathrm{~V})$ in order to retain a reasonable image size. The voltage ratio between the repeller and extractor plate was carefully optimized at the beginning of the experiment to obtain the best velocity mapping of the $\mathrm{H}_{2} \mathrm{O}^{+}$ions on the MCP detector.

\section{B. Quantum scattering calculations}

The quantum scattering calculations employed the PES obtained by Patkowski et al. [14] Although there are (slightly) more recent $\mathrm{He}-\mathrm{H}_{2} \mathrm{O}$ PES available, $[15,16]$ work by Yang and Stancil showed that all these PES showed some differences only at very low collision energies, [17] which are not relevant to this work. The close-coupling calculations were performed using the 4.4 version of the HIBRIDON program,[18] which uses 
the improved log-derivative propagator by Manolopoulos and co-workers. $[19,20]$ The wavefunction was propagated between 3.5 and $130 a_{0}$ using a step of $0.04 a_{0}$. We followed closely the methodology presented by Dagdigian and Alexander in their study of $\mathrm{He}+\mathrm{H}_{2} \mathrm{O}$ depolarizing collisions.[21] The $\mathrm{H}_{2} \mathrm{O}$ molecule was described as a rigid rotor asymmetric top with rotational constants $A=27.881 \mathrm{~cm}^{-1}, B=14.522 \mathrm{~cm}^{-1}$, and $C=$ $9.278 \mathrm{~cm}^{-1}$.[21] A maximum value of total angular momentum $\left(J_{\max }=150\right)$, a maximum value of the rotational quantum number of the $\mathrm{H}_{2} \mathrm{O}$ molecule $\left(j_{\max }=15\right)$, and all channels up to $2100 \mathrm{~cm}^{-1}$ were employed in order to converge the cross sections. The values of the energy levels of all the open channels obtained from our calculations are compared in Table 1 with the IUPAC recommended values by Tennyson et al.[22] The reduced mass of the $\mathrm{He}-\mathrm{H}_{2} \mathrm{O}$ was set equal to $3.27481925 \mathrm{amu}$. Both elastic and inelastic cross sections were converged, and the calculated DCS were obtained with resolution of $1^{\circ}$.

In order to test our calculations, we compared the ICS results with those from previous studies. Yang et al. [23] calculated ICS using various PESs and they found quite different results compared with those by Yang et al. [12] using the same PESs. In Table II, we compare our results with previous calculations. Results from this work are almost identical with the most recent work. [23] The disagreement with the earlier study [12] was due to some problems in the expansion of the PES used in the close-coupling calculations. This disagreement has now been resolved as shown in Table 2, where using MOLSCAT [24] and a refitted expansion (using 29 terms) of the PES by Hodges et al. [15] provides similar results with the HIBRIDON calculations using the PES by Patkowski et al. [14]. All the subsequent calculations were performed using HIBRIDON. 
Table 1. Rotational energy levels $\left(\mathrm{cm}^{-1}\right)$ of ortho- and para- $\mathrm{H}_{2} \mathrm{O}$ obtained from calculations from this work, and from the IUPAC recommended values obtained by Tennyson et al.[22] Values rounded to three decimal places are presented for all channels that are open at the highest collision energy $\left(763.17 \mathrm{~cm}^{-1}\right)$ employed in the experiments.

\begin{tabular}{|c|c|c|c|c|c|c|c|}
\hline$N$ & $J_{K a K c}$ & Energ & & $N$ & $J_{K a K c}$ & Ener & \\
\hline & & & $\begin{array}{l}\text { Tennyson } \\
\text { et al. [22] }\end{array}$ & & & This work & $\begin{array}{l}\text { Tennyson } \\
\text { et al. [22] }\end{array}$ \\
\hline 1 & $1_{01}$ & 23.800 & 23.794 & 1 & $0_{00}$ & 0.000 & 0.000 \\
\hline 2 & $1_{10}$ & 42.403 & 42.372 & 2 & $1_{11}$ & 37.159 & 37.137 \\
\hline 3 & $2_{12}$ & 79.515 & 79.496 & 3 & $2_{02}$ & 70.134 & 70.091 \\
\hline 4 & $22_{21}$ & 135.324 & 134.902 & 4 & $2_{11}$ & 95.247 & 95.176 \\
\hline 5 & $3_{03}$ & 136.893 & 136.762 & 5 & 20 & 136.590 & 136.164 \\
\hline 6 & $3_{12}$ & 173.601 & 173.366 & 6 & $3_{13}$ & 142.334 & 142.278 \\
\hline 7 & $3_{21}$ & 212.631 & 212.156 & 7 & $3_{22}$ & 206.724 & 206.301 \\
\hline 8 & $4_{14}$ & 224.999 & 224.838 & 8 & $4_{04}$ & 222.316 & 222.053 \\
\hline 9 & $3_{30}$ & 287.541 & 285.419 & 9 & $4_{13}$ & 276.104 & 275.497 \\
\hline 10 & $4_{23}$ & 300.925 & 300.362 & 10 & $3_{31}$ & 287.344 & 285.219 \\
\hline 11 & $5_{05}$ & 325.797 & 325.348 & 11 & $4_{22}$ & 316.584 & 315.780 \\
\hline 12 & $4_{32}$ & 384.591 & 382.517 & 12 & $5_{15}$ & 326.980 & 326.625 \\
\hline 13 & $5_{14}$ & 400.678 & 399.458 & 13 & $4_{31}$ & 385.926 & 383.843 \\
\hline 14 & $6_{16}$ & 447.909 & 447.252 & 14 & $5_{24}$ & 417.127 & 416.209 \\
\hline 15 & $5_{23}$ & 448.106 & 446.511 & 15 & $6_{06}$ & 447.425 & 446.697 \\
\hline 16 & $4_{41}$ & 494.695 & 488.108 & 16 & $4_{40}$ & 494.720 & 488.134 \\
\hline 17 & $5_{32}$ & 511.242 & 508.812 & 17 & $5_{33}$ & 506.283 & 503.968 \\
\hline 18 & $6_{25}$ & 554.451 & 552.911 & 18 & $6_{15}$ & 544.927 & 542.906 \\
\hline 19 & $7_{07}$ & 587.386 & 586.244 & 19 & $7_{17}$ & 587.575 & 586.479 \\
\hline 20 & $5_{41}$ & 616.717 & 610.341 & 20 & $6_{24}$ & 605.752 & 602.773 \\
\hline 21 & $6_{34}$ & 651.983 & 648.979 & 21 & $5_{42}$ & 616.493 & 610.114 \\
\hline 22 & $7_{16}$ & 707.177 & 704.214 & 22 & $6_{33}$ & 665.057 & 661.549 \\
\hline 23 & $8_{18}$ & 745.873 & 744.163 & 23 & $7_{26}$ & 712.058 & 709.608 \\
\hline 24 & $5_{50}$ & 757.745 & 742.076 & 24 & $8_{08}$ & 745.801 & 744.163 \\
\hline 25 & $6_{43}$ & 763.340 & 756.725 & 25 & $5_{51}$ & 757.742 & 742.073 \\
\hline 26 & $7_{25}$ & 787.401 & 782.410 & 26 & $6_{42}$ & 764.413 & 757.780 \\
\hline
\end{tabular}


Table 2. Comparison between state-to-state theoretical inelastic cross sections $\left(\AA^{2}\right)$ at $429 \mathrm{~cm}^{-1}$ obtained from previous and current work using the PES by Hodges et al. [15] (columns 2 and 3, respectively) and using the PES by Patkowski et al. [14] (columns 4 and 5).

\begin{tabular}{|c|c|c|c|c|}
\hline$J_{K a K c} \rightarrow J_{K_{a}^{\prime} K_{c}^{\prime}}^{\prime}$ & Yang et al. (2010) [12] & This work & Yang et al. (2013) [23] & This work \\
\hline $0_{00} \rightarrow 1_{11}$ & 10.1800 & 7.978 & 8.9564 & 8.9650 \\
\hline $0_{00} \rightarrow 2_{02}$ & 1.2620 & 2.437 & 2.2541 & 2.2613 \\
\hline $0_{00} \rightarrow 2_{11}$ & 0.0070 & 0.0091 & 0.0077 & 0.0077 \\
\hline $0_{00} \rightarrow 2_{20}$ & 1.2500 & 0.713 & 0.8267 & 0.8397 \\
\hline $0_{00} \rightarrow 3_{13}$ & 1.2020 & 1.951 & 1.8413 & 1.7955 \\
\hline $0_{00} \rightarrow 3_{22}$ & 0.0010 & 0.0005 & 0.0004 & 0.0004 \\
\hline $1_{11} \rightarrow 2_{02}$ & 3.1150 & 3.146 & 3.3162 & 3.2928 \\
\hline $1_{11} \rightarrow 2_{11}$ & 1.7120 & 2.775 & 2.6612 & 2.6600 \\
\hline $1_{11} \rightarrow 2_{20}$ & 3.3880 & 2.293 & 2.7048 & 2.6955 \\
\hline $1_{11} \rightarrow 3_{13}$ & 0.7320 & 0.968 & 1.0468 & 1.0398 \\
\hline $1_{11} \rightarrow 3_{22}$ & 0.2050 & 0.282 & 0.2827 & 0.2838 \\
\hline $1_{01} \rightarrow 1_{10}$ & 5.2720 & 4.221 & 4.7021 & 4.7053 \\
\hline $1_{01} \rightarrow 2_{12}$ & 5.4290 & 4.670 & 5.1073 & 5.0909 \\
\hline $1_{01} \rightarrow 2_{21}$ & 0.8790 & 0.493 & 0.5636 & 0.5756 \\
\hline $1_{01} \rightarrow 3_{03}$ & 0.7180 & 1.051 & 1.0213 & 1.0136 \\
\hline $1_{01} \rightarrow 3_{12}$ & 0.0960 & 0.119 & 0.1119 & 0.1093 \\
\hline $1_{01} \rightarrow 3_{21}$ & 0.4770 & 0.400 & 0.4334 & 0.4366 \\
\hline $1_{01} \rightarrow 4_{14}$ & 0.5450 & 0.655 & 0.6187 & 0.6075 \\
\hline
\end{tabular}


In order to compare our theoretical and experimental results, we had to calculate separately ICS and DCS for ortho- $\mathrm{H}_{2} \mathrm{O}$ (for $1_{01}$ and for $1_{10}$ initial levels) and para- $\mathrm{H}_{2} \mathrm{O}$ (for $\mathrm{O}_{00}$ and for $1_{11}$ initial levels). The theoretical ICSs for the lowest experimental collision energy of $381.34 \mathrm{~cm}^{-1}$ is shown in Fig. 3. The elastic cross sections are not shown in Fig. 3 because they are much larger (between 60.05 and $62.39 \AA^{2}$ ) than the inelastic ones. The theoretical DCSs for $\mathrm{He}+\mathrm{H}_{2} \mathrm{O}\left(\mathrm{O}_{00}\right)$ at $E_{\text {col }}=381.34 \mathrm{~cm}^{-1}$ are shown in Fig. 4.

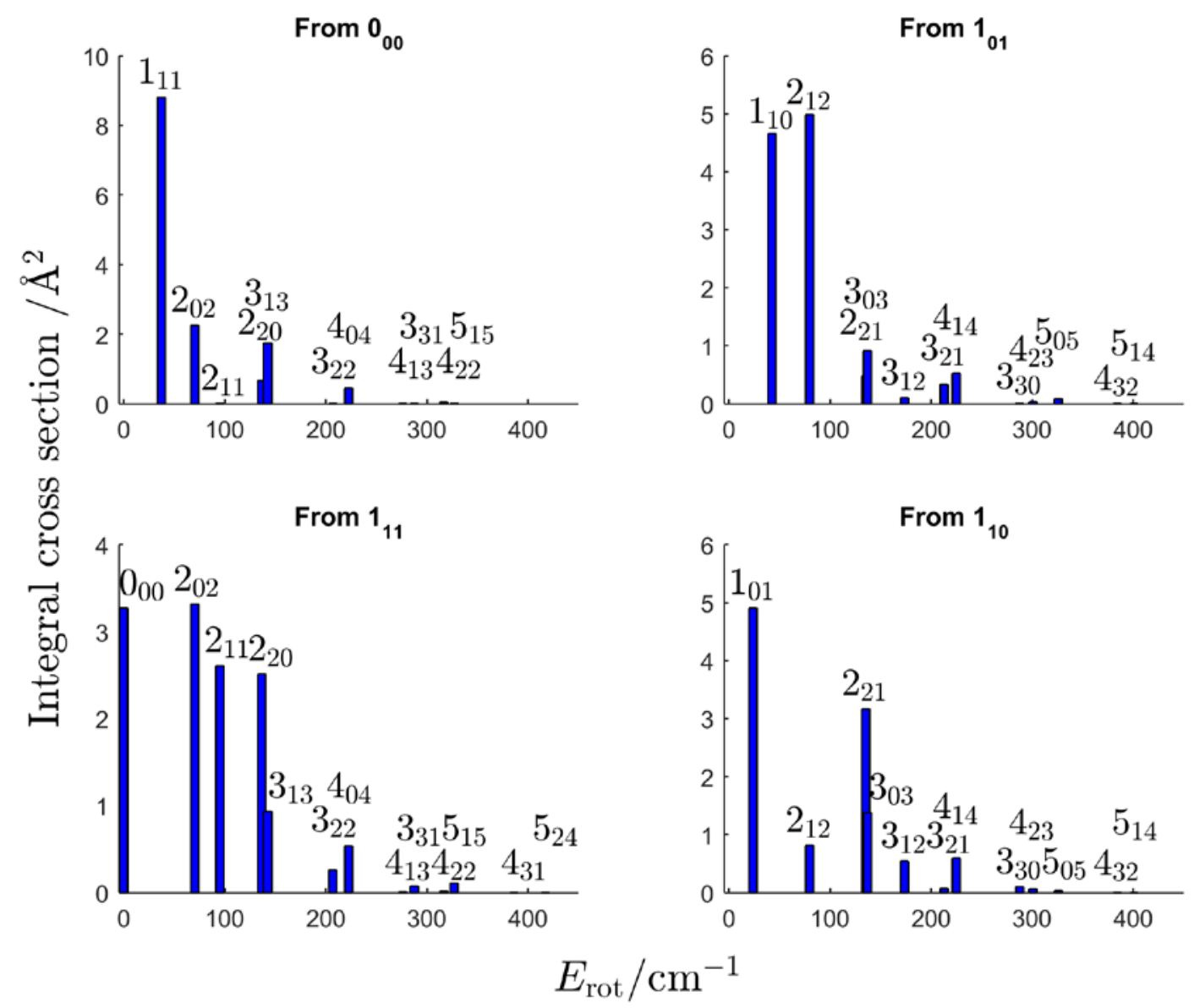

Figure 3: Close-coupling quantum mechanical state-to-state inelastic scattering cross sections for $\mathrm{He}+\mathrm{H}_{2} \mathrm{O}$ at $E_{\text {col }}=381.34 \mathrm{~cm}^{-1}$ plotted versus $\mathrm{H}_{2} \mathrm{O}$ rotational energy. The initial $\mathrm{H}_{2} \mathrm{O}$ rotational level is depicted on the title of each graph, and the final rotational level is shown near the corresponding bar. 

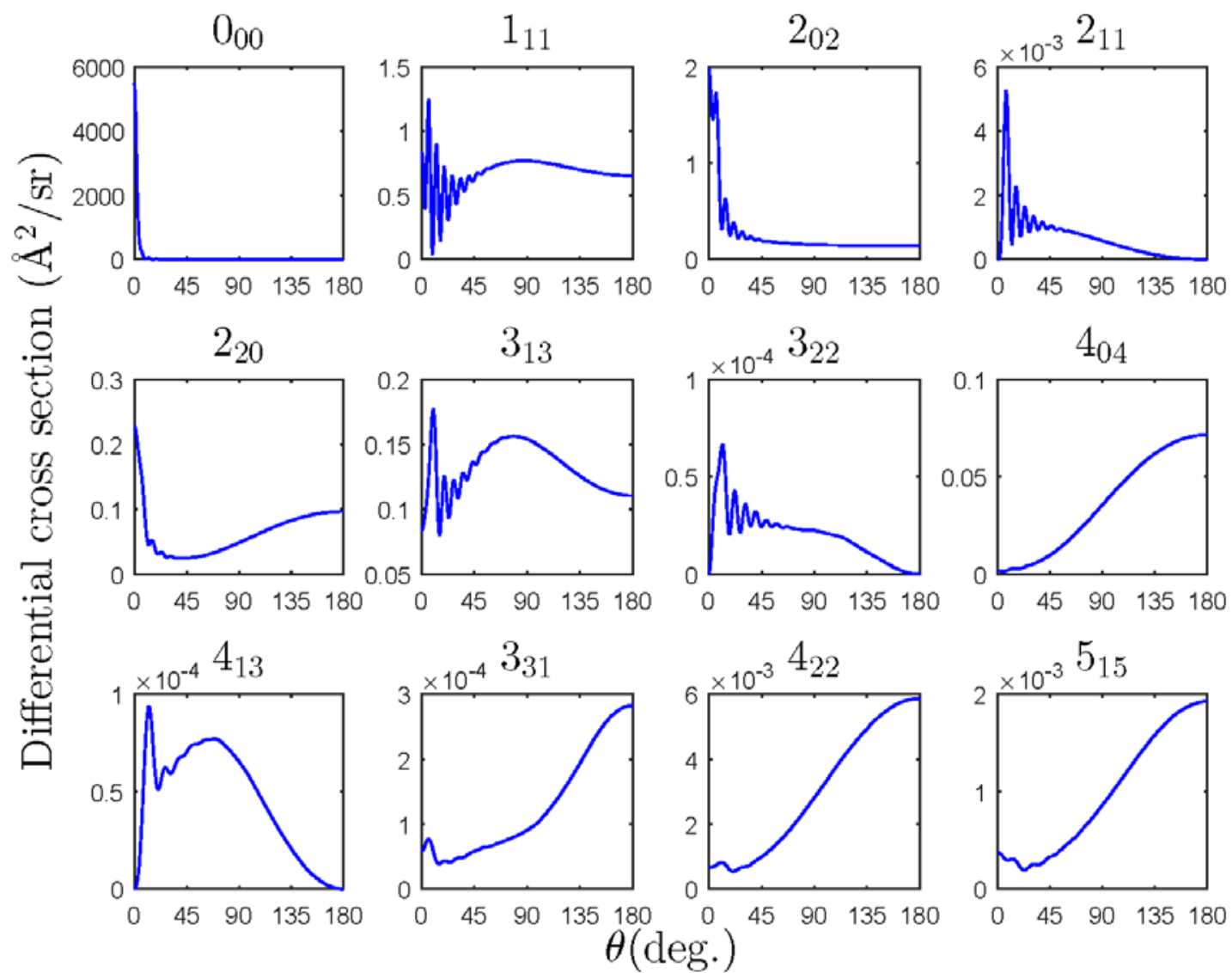

Figure 4: Close-coupling quantum mechanical differential cross sections (DCSs) for transitions out of $\mathrm{He}+\mathrm{H}_{2} \mathrm{O}$ $\left(0_{00}\right)$ at $E_{\mathrm{col}}=381.34 \mathrm{~cm}^{-1}$. The final $\mathrm{H}_{2} \mathrm{O}$ rotational levels are shown in the title of each graph, and the DCSs are presented in increasing rotational energy of the final level. 


\section{Results}

A series of raw $\mathrm{H}_{2} \mathrm{O}^{+}$images taken for five different crossing angles is shown in Fig. 5 . In these false-color images, the intensity was higher in the forward scattering direction. A sketch of the relevant velocity vectors for a typical velocity mapped image of $\mathrm{H}_{2} \mathrm{O}$ scattered by $\mathrm{He}$ at a collision energy of $694.47 \mathrm{~cm}^{-1}$ is also shown in Fig. 5. In order to measure integral ICSs or DCSs in a crossed beam experiment using focused nanosecond pulsed dye laser, we have a large temporal overlap of the two molecular beams, and a laser pulse of a short duration time in a finite detection volume. When we varied the angle between the two molecular beams, both the center of mass and the relative velocity in the lab frame were altered. As a consequence, the density distribution (enhanced for slow molecules in the lab frame), in the corresponding image (Fig. 5) was also altered. A density-to-flux transformation was needed to extract the DCS from the velocity mapped images of scattered molecules. The procedure to obtain this density-to-flux transformation has been described in some of our previous papers.[12, 13, 25] 


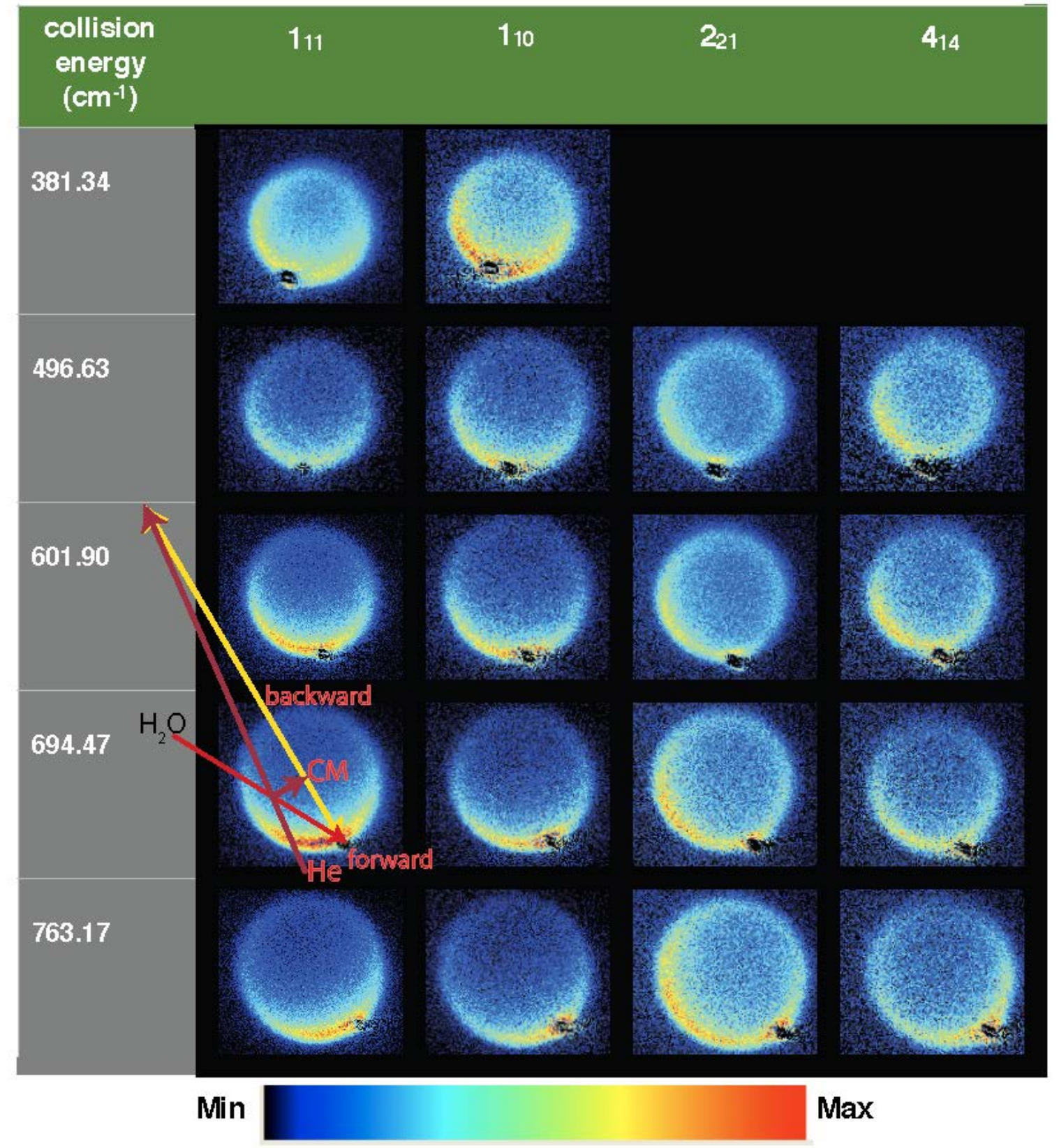

Figure 5: Experimental raw velocity mapped images for scattering of $\mathrm{H}_{2} \mathrm{O}$ with $\mathrm{He}$. The final $\mathrm{H}_{2} \mathrm{O}$ rotational levels are indicated on the top line, and the collision energies are shown on the left column. The linear intensity scale for the signal is shown on the bottom side. The directions of the $\mathrm{He}$ and $\mathrm{H}_{2} \mathrm{O}$ beams, and their center-of-mass relative velocity are sketched for the final $\mathrm{H}_{2} \mathrm{O} 1_{11}$ level at a collision energy of $694.47 \mathrm{~cm}^{-1}$. 
Differential cross sections of $\mathrm{H}_{2} \mathrm{O}$ extracted from the experimental images (Fig. 5) are shown in Fig. 6. The four columns in Fig. 6 contain the DCS curves for the four rotational transitions. In order to aid comparison, all the DCSs from the same rotational transition are plotted on the same column in increasing collision energy order. The experimental results are normalized to the theoretical values, which have been averaged over the initial populations. The $0-5^{\circ}$ range was not taken into account in the normalization because the experimental data are less reliable in the extreme forward direction. Taking a look at the DCS into the lowest $1_{11}$ rotational level of $\mathrm{H}_{2} \mathrm{O}$, we see a significant change from sideways scattering at $E_{\mathrm{col}}=381.34 \mathrm{~cm}^{-1}$ to forward scattering at $E_{\mathrm{col}}=763.17 \mathrm{~cm}^{-1}$. A similar collision energy dependence is observed for the highest $4_{14}$ level (especially in the theoretical calculations), but the trend is not clearly observed for the intermediate $1_{10}$ and $2_{21}$ level. DCSs for excitations to the lowest final $1_{11}$ and $1_{10}$ rotational levels are much more forward than for excitations to the highest final $2_{21}$ and $4_{14}$ levels. This final level dependence of the DCS is more pronounced at the lowest collision energy. 

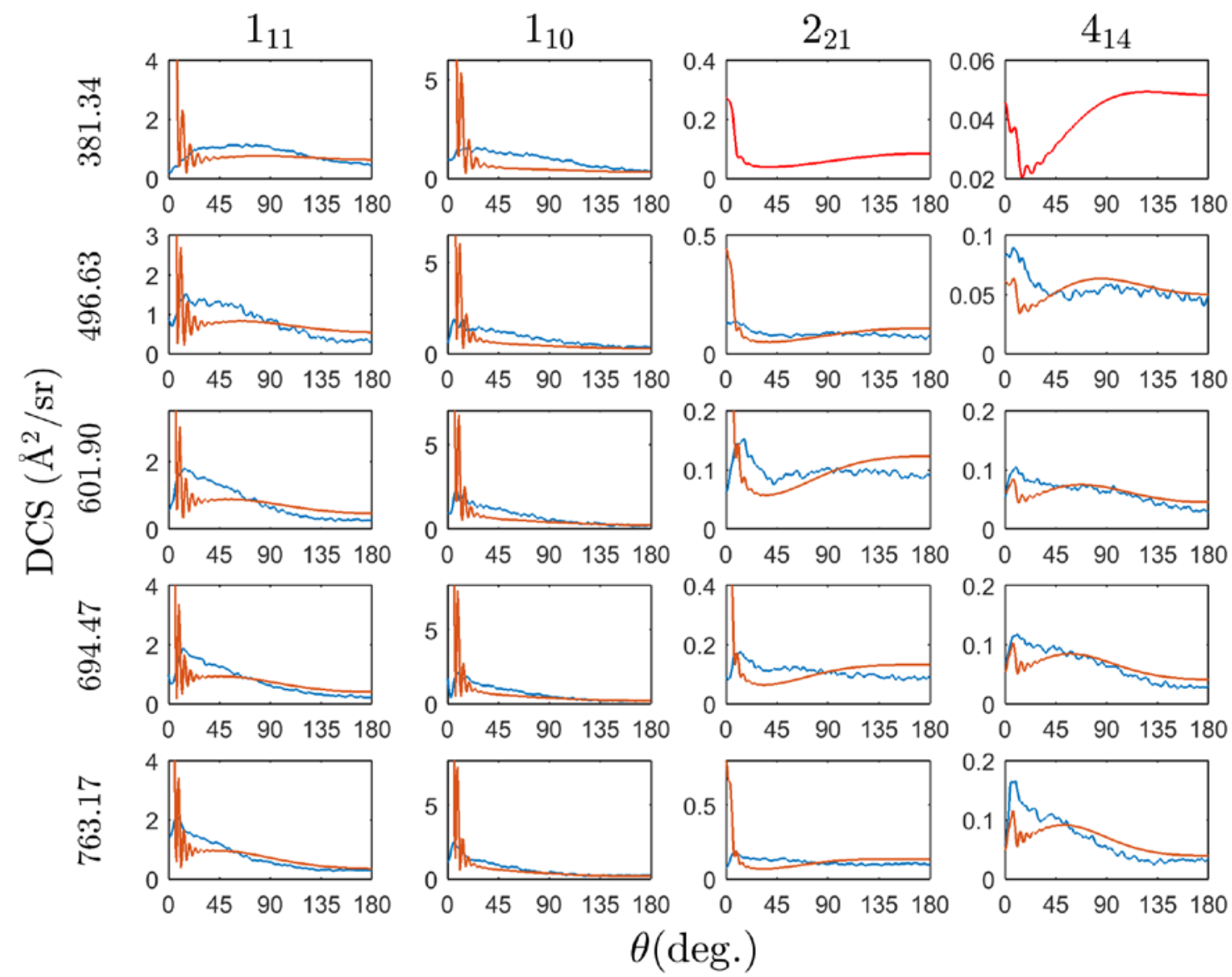

$\theta$ (deg.)

Figure 6: Differential cross sections for scattering of $\mathrm{H}_{2} \mathrm{O}$ by He for $1_{11}, 1_{10}, 2_{21}$ and $4_{14}$ final rotational levels of $\mathrm{H}_{2} \mathrm{O}$ at collision energies of $381.34,496.63,601.90,694.47$ and $763.17 \mathrm{~cm}^{-1}$. The final $\mathrm{H}_{2} \mathrm{O}$ levels are shown at the top of each column, and the collision energy (in $\mathrm{cm}^{-1}$ ) at the left of each row. The theoretical values (red) have been averaged over the initial experimental populations, and the experimental values (blue) have been normalized to the averaged theoretical values neglecting the $0-5^{\circ}$ range. 
A DCS can usually be described as isotropic, forward, sideways or backward. To the best of our knowledge, however, there is not yet a quantitative way to define the 'forwardness' or 'backwardness' of a DCS. For this reason, we devised the forward-to-backward ratio, FBR, as:

$$
F B R=\frac{\int_{0}^{90} w(x) D C S \sin \theta d \theta}{\int_{90}^{180} w(x) D C S \sin \theta d \theta}
$$

In eq. (3), we multiply the DCS by $\sin \theta d \theta$ as this is necessary to provide the ICS. The weight function, $w(x)$, can be any known function, i.e. unit, linear, cosine or depend on the particular experiment and the associated uncertainties. In Fig. 7, we show the theoretical DCS for the $4_{14}$ final level at $381.34 \mathrm{~cm}^{-1}$. The FBR ratios for unit $w(x)$ are shown on the Fig. 7, and have been tabulated for all the available DCS functions in Table 3. There are some obvious benefits in using the FBR ratios. For example, in order to compare the 'forwardness' of two DCS, we need only two numbers, the ratios, instead of all the DCS points. Also, it is straightforward to study quantitatively the collision energy dependence of the 'forwardness' of a DCS by looking at the numbers in a table. For example, we see in Table 3 that the DCS becomes more forward by increasing the collision energy with only few exceptions. If there is no particular reason, using the unit function seems to be the preferred choice. On the other hand, multiplying the DCS by linear or cosine or other functions might be useful if one needs to express it as a Legendre or other mathematical series. 
a)

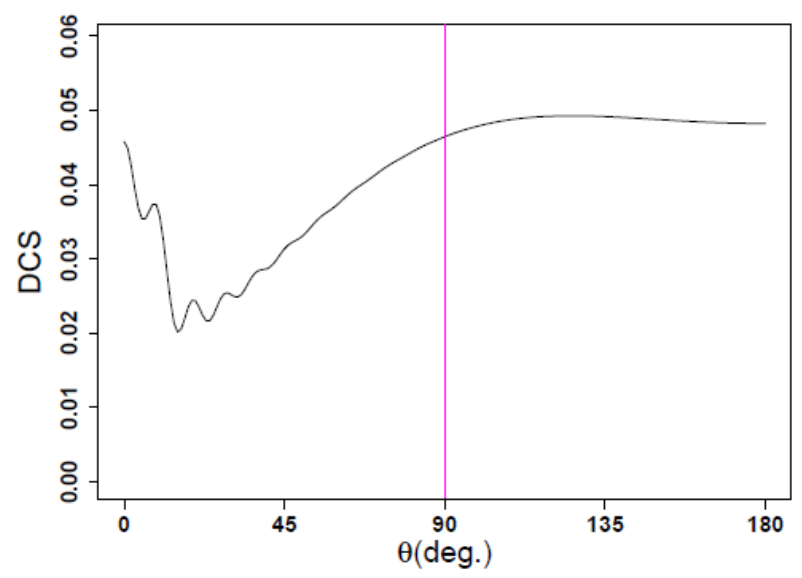

c)

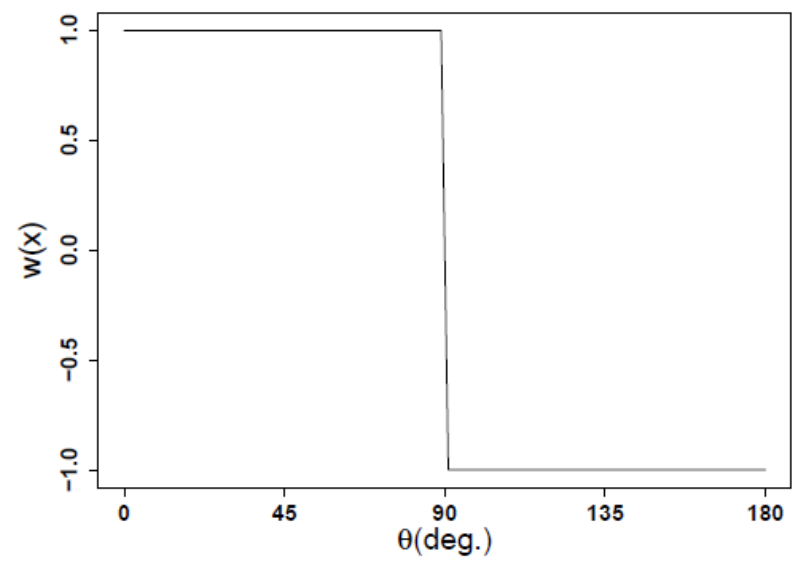

b)

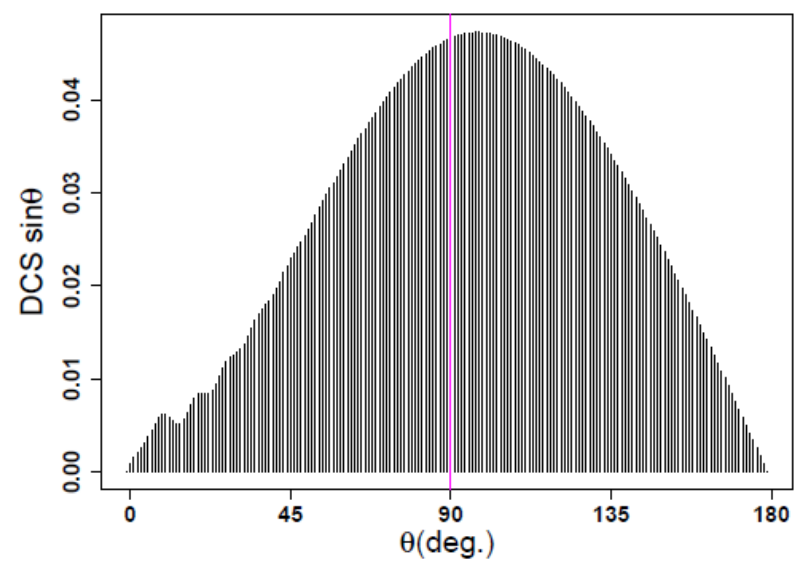

d)

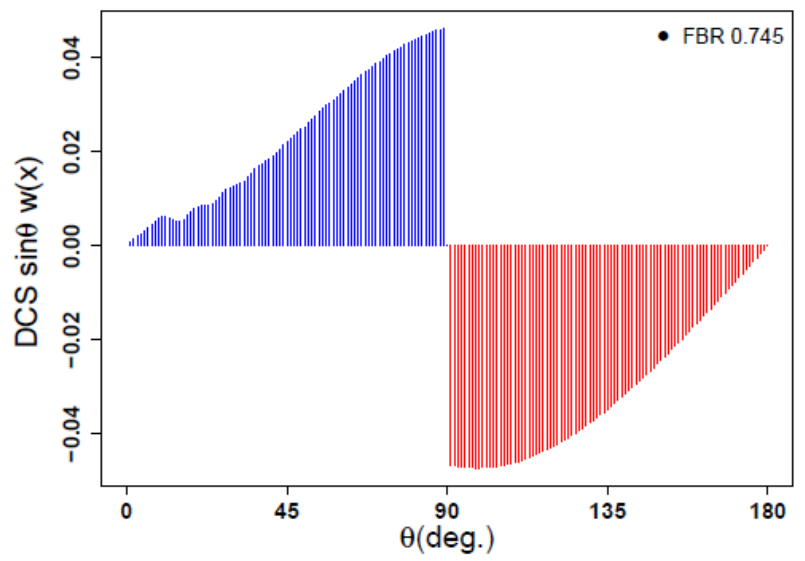

Figure 7: (a) DCS function for the $4_{14}$ final level at $381.34 \mathrm{~cm}^{-1}$. (b) DCS multiplied by the $\sin \theta$ function. (c) The unit weight function. (d) DCS multiplied by the weight function and the calculated forward-to-backward ratio, FBR. 
Table 3. Comparison between theoretical and normalized experimental forward-to-backward ratios (FBR)

(eq.3). The results are obtained using the unit weight function $w(x)$.

\begin{tabular}{|c|c|c|c|}
\hline $\begin{array}{c}\text { Final level } \\
J^{\prime} K_{a}^{\prime} K_{c}^{\prime}\end{array}$ & $\begin{array}{c}E_{\mathrm{col}} \\
\left(\mathrm{cm}^{-1}\right)\end{array}$ & FBR (expt) & FBR (theor.) \\
\hline $1_{11}$ & 381.34 & 1.35 & 1.32 \\
\hline $1_{11}$ & 496.63 & 2.13 & 1.47 \\
\hline $1_{11}$ & 601.90 & 2.79 & 1.66 \\
\hline $1_{11}$ & 694.47 & 2.67 & 1.86 \\
\hline $1_{11}$ & 763.17 & 2.52 & 2.02 \\
\hline $1_{10}$ & 381.34 & 1.76 & 3.24 \\
\hline $1_{10}$ & 496.63 & 2.00 & 3.39 \\
\hline $1_{10}$ & 601.90 & 2.80 & 3.65 \\
\hline $1_{10}$ & 694.47 & 3.07 & 3.95 \\
\hline $1_{10}$ & 763.17 & 2.71 & 4.20 \\
\hline 221 & 381.34 & - & 0.672 \\
\hline 221 & 496.63 & 1.02 & 0.670 \\
\hline 221 & 601.90 & 1.00 & 0.682 \\
\hline 221 & 694.47 & 1.24 & 0.700 \\
\hline 221 & 763.17 & 1.23 & 0.719 \\
\hline $4_{14}$ & 381.34 & - & 0.747 \\
\hline $44_{14}$ & 496.63 & 1.02 & 0.968 \\
\hline $4_{14}$ & 601.90 & 1.41 & 1.21 \\
\hline $4_{14}$ & 694.47 & 1.93 & 1.44 \\
\hline $4_{14}$ & 763.17 & 2.41 & 1.61 \\
\hline
\end{tabular}

The integral cross sections for the final $\mathrm{H}_{2} \mathrm{O} 1_{11}$ level are shown in Fig. 8. The ICSs at each collision energy are obtained by integrating the angular distribution of the scattered $\mathrm{H}_{2} \mathrm{O}$ molecule measured using velocity map imaging. Note that the collision energies shown in Fig. 8 are not the same with those in Fig. 6 . All the 
measurements shown in Fig. 8 were obtained on the same day under the same conditions. Repeated experiments were performed to ensure that the molecular beam densities and the laser power were unchanged when we varied the collision energy. The ICSs include some contribution from elastic scattering as well, but this should be minimal considering the rotationally cold $\mathrm{H}_{2} \mathrm{O}$ beam ( $3 \%$ in the $1_{11}$ level before the collision). We note that the ICS decreases at higher collision energies.

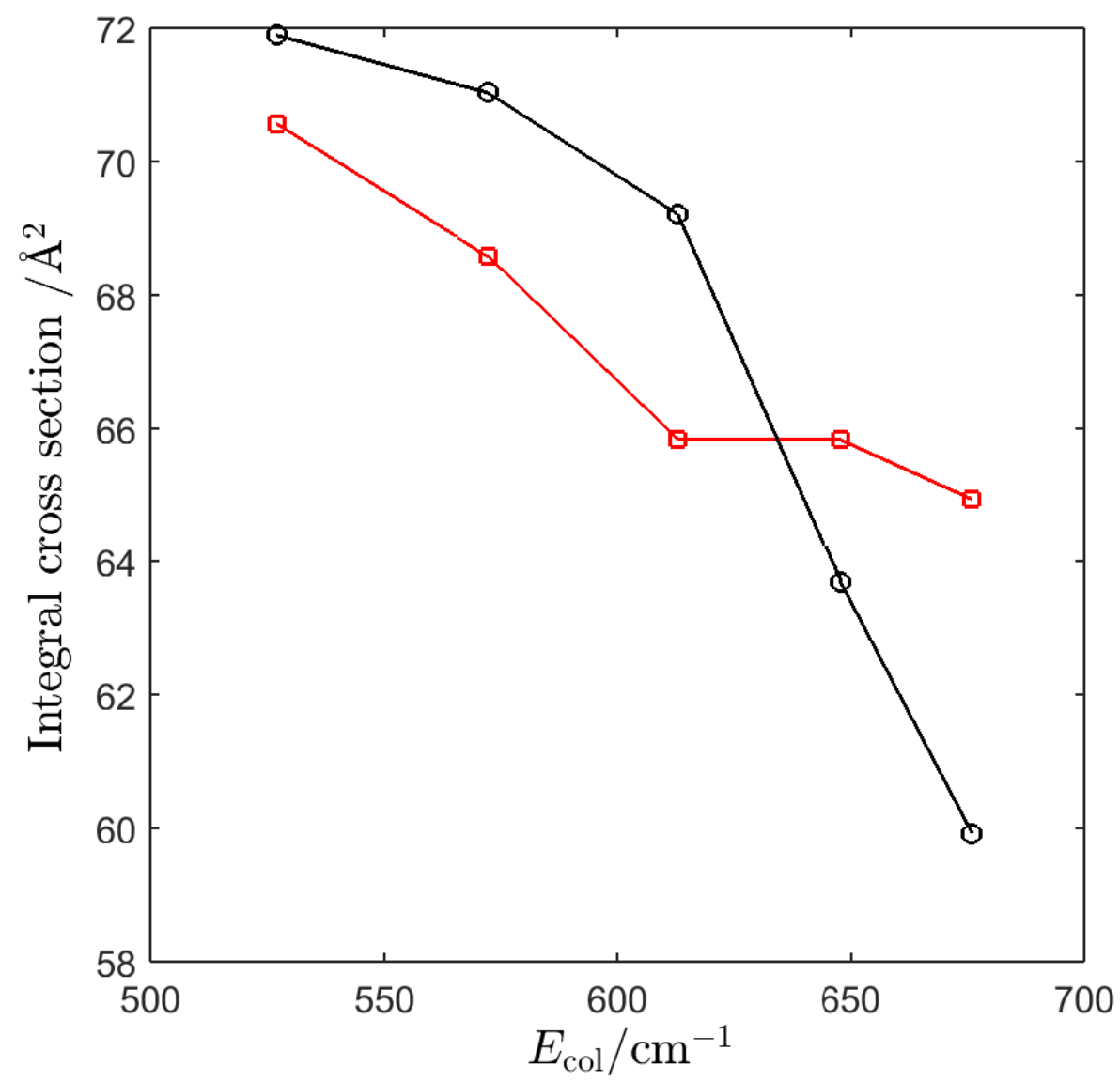

Figure 8: Comparison between the experimental (circles) and theoretical (squares) total (elastic and inelastic) cross section of $\mathrm{He}+\mathrm{H}_{2} \mathrm{O}$ collisions into the $\mathrm{H}_{2} \mathrm{O} 1_{11}$ final rotational level at collision energies between 500 and $700 \mathrm{~cm}^{-1}$. 


\section{Rotational rainbow scattering}

If inelastic collisions are dominated by repulsive interactions, it should be possible to observe rotational rainbows, which are sensitive probes of the potential energy surface anisotropy. In the simplest case of the scattering of a heteronuclear diatomic molecule from an atom and when neglecting of attractive forces, the interaction potential can be approximated by a rigid ellipsoid (Fig. 9).[26-28] The semi-major and semi-minor axes of the ellipse are denoted A and B, respectively. As shown in Fig. 9, the center-of-mass of the molecule, $\mathrm{C}$, and the centre of the ellipsoid, $\mathrm{O}$, differ by $\delta$. In this 2D model, only the component of the initial momemum $p_{i}$ that is perpendicular to the ellipsoid at the point of impact (parallel to the $n$ axis) can induce rotation of the ellipsoid. The angle between $p_{i}$ and $n$ is called $\varphi$, and the angle between the final momentum $p_{f}$ and $n$ is called $\varphi_{R}$. 


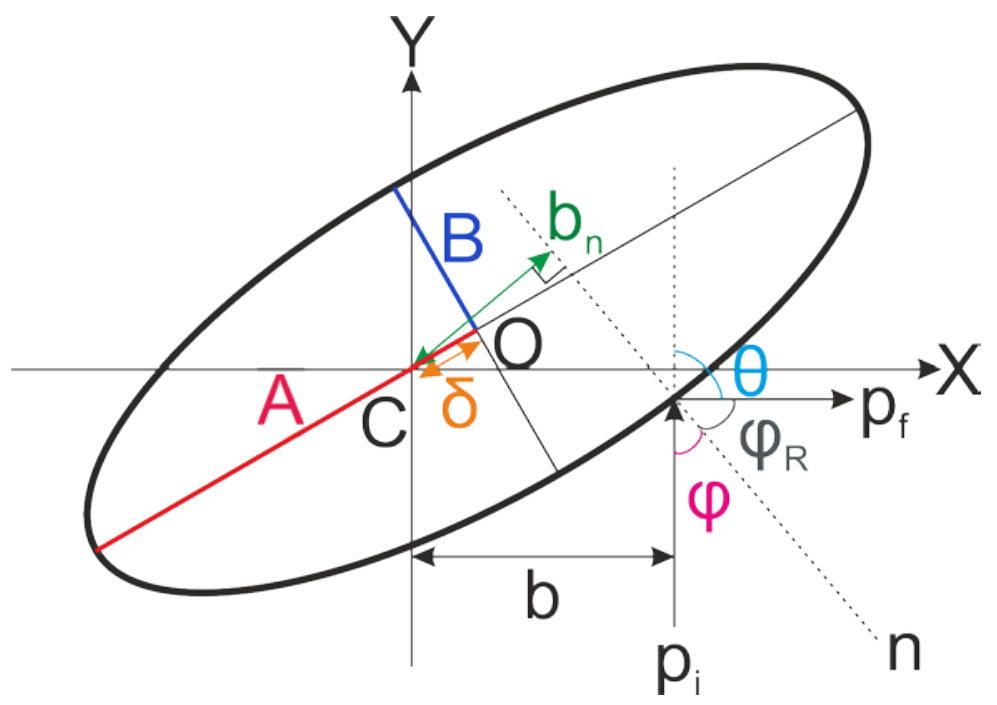

Figure 9: Two-dimensional heteronuclear diatomic molecule-atom collision geometry. The definition of collision parameters is adapted from Ref. [27]. A and B represent the two semi-axes of the ellipsoid, and $O$ is the centre of the ellipsoid. The incident and final momentum of the colliding atom are denoted $p_{i}$ and $p_{f}$, respectively. The $n$ axis points perpendicular to the ellipsoid hard shell surface. $b$ is the impact parameter, and $\theta$ represents the deflection or scattering angle. The effective impact parameter, $b_{n}$, is the shortest distance between the center of mass of the ellipsoid, $\mathrm{C}$, and the $n$ axis. 
It is pictorially obvious that the maximum torque transferred to the ellipsoid depends on the impact point of the approaching atom. This position of the impact point can be calculated and related to the effective impact parameter, $b_{\mathrm{n}}$, which is the shortest distance between $\mathrm{C}$ and $\mathrm{n}$, and determines the magnitude of the torque. This value can in turn be connected to the rainbow angle, $\theta_{R}$, and to the final angular momentum, $J_{f}$, by [28]

$$
\sin \left(\frac{\theta_{\mathrm{R}}}{2}\right)=\frac{J_{f}}{2 P_{i} b_{\max }}
$$

where $p_{i}$ is the incident momentum of the colliding atom, and $b_{\max }$ is the maximum of $b_{\mathrm{n}}$ which leads to the rotational rainbow angle. For an elliptic homonuclear hard sphere, $b_{\max }=A-B$, i.e. is equal to the difference between the semi-major and semi-minor axes of the ellipsoid.[28] For the heteronuclear case, $b_{\max }=A-B \pm \delta(A / A+B)^{\frac{1}{2}}$ in the case of small center shift compared with the half-axes difference.[27] This is of course a classical picture which neglects, as was already mentioned, the attractive part of the potential. The influence of the attractive part on the position of the rotational rainbow has been derived in model calculations.[29] From equation 4, for scattering into a low $J_{f}$ level, the rotational rainbow peak is expected at relatively low scattering angles.[12] As the rainbow angle is inversely proportional to $p_{i}$, the energy dependence would cause a decrease in the position of the rainbow angle with an increase of collision energy. Therefore, the rotational rainbow angle positions will be determined as a result of competition between the $b_{\max }$ and the collision energy.

In this discussion so far, we considered a simple heteronuclear diatomic molecule ellipsoid to describe the concept of rotational rainbows. For asymmetric top collisions of the $\mathrm{H}_{2} \mathrm{O}-\mathrm{He}$ system, this model needs to be extended using separate ellipsoids for each rotation axis.[7, 12] For this reason, we cut the Patkowski PES through three planes each of which points perpendicular to one of the three principal axes of $\mathrm{H}_{2} \mathrm{O}$. For a given total angular momentum

$J$ of $\mathrm{H}_{2} \mathrm{O}$, the three values of $K_{a} \approx J$ (e.g. $1_{10}$ and $2_{21}$ ), $K_{a} \approx K_{c}$ (e.g. $1_{11}$ ) and $K_{c} \approx J$ (e.g. $4_{14}$ ) correspond to rotation around the $a, b$ and $c$ axis, respectively.[11] The lower, intermediate and higher rainbow 
angles are expected for rotation around the $c, b$ and $a$ axes due to the larger anisotropy. [7, 12] The values of the anisotropic parameters, and of the maxima effective impact parameters for $\mathrm{He}+\mathrm{H}_{2} \mathrm{O}$ at various experimental collision energies are shown in Table 4. 
Table 4. Maximum effective impact parameters for $\mathrm{He}+\mathrm{H}_{2} \mathrm{O}$ collisins at various collision energies using the PES by Patkowski et al. [14].

\begin{tabular}{|c|c|c|c|}
\hline \multirow{2}{*}{$\begin{array}{l}\text { Energy for } \\
\text { equi-potential } \\
\text { cut }\left(\mathrm{cm}^{-1}\right)\end{array}$} & \multicolumn{3}{|c|}{ Maximum $b_{n} / \AA$} \\
\hline & Rotation around a-axis & Rotation around $b$-axis & Rotation around c-axis \\
\hline \multirow[t]{3}{*}{381} & 0.074 & 0.065 & 0.434 \\
\hline & & & 0.268 \\
\hline & 0.015 & & 0.152 \\
\hline \multirow[t]{3}{*}{497} & 0.069 & 0.053 & 0.428 \\
\hline & & & 0.270 \\
\hline & 0.015 & & 0.144 \\
\hline \multirow[t]{3}{*}{602} & 0.064 & 0.045 & 0.424 \\
\hline & & & 0.275 \\
\hline & 0.014 & & 0.139 \\
\hline \multirow[t]{3}{*}{694} & 0.060 & 0.040 & 0.421 \\
\hline & & & 0.280 \\
\hline & 0.014 & & 0.136 \\
\hline \multirow[t]{3}{*}{763} & 0.059 & 0.041 & 0.418 \\
\hline & & & 0.283 \\
\hline & 0.014 & & 0.134 \\
\hline
\end{tabular}


The general result of the present paper is to demonstrate the energy dependence for several single rotational transitions. These are $4_{14}$ (rotation around the c-axis), $1_{11}$ (rotation around the b-axis), $1_{10}$ and $2_{21}$ (rotation around the $a$-axis). The agreement between experiment and theoetical calculations is quite reasonable. The rotational rainbows are clearly observed for $4_{14}$ as sort of second peak. For $1_{11}$ the observation starts with an insolated peak and also moves as a sort of shoulder to smaller angles. The $1_{10}$ excitation consists only of forward scattering and also for $2_{21}$ the rainbows are not very pronounced. Thus we concentrate on the rotations around the $c$-axis and $b$-axis. For the final rotational levels $4_{14}$ and $1_{11}$, the peaks move in general to smaller angles with increasing collision energy. This behavior can easily explained by the rotational rainbow model of Eq. (4). The rotational rainbow angle is inversely proportional to the square root of the energy. This definitely descibes the general trend. But we have also to consider the anisotropy parameter $b_{\text {max }}$ which might contain some energy dependence. As can be seen by the results of Table 4 , the effect slightly increases the angle but it is pretty small. Thus the general tendency of the peaks at the larger energies is correctly explained by the model.

We now have to explain the shift of the peaks, say at the energy of $496.6 \mathrm{~cm}^{-1}$, to smaller values going from the $c$-axis to the $b$-axis. A look at the $b_{\max }$ values of Table 4 demonstrates just the opposite behavior. The $b_{\max }$ values of the $c$-axis are much larger than those of the $b$-axis and should therefore shift the rainbow angle to smaller values. But here we have to take into account that also the final rotational state $J_{\max }$ enters the formula. It is a factor of 4 higher for the $c$-axis than for the $b$-axis which partly compensates the effect of $b_{\max }$ just mentioned. We do observe such a trend also for the rotation in different axes when we compare theoretical values of DCS into the same final state $2_{02}, 2_{11}$ and $2_{20}$ in Fig. 4 . They correspond to the $c$-axis, $b$-axis, and $a$-axis, respectively, and exhibit as predicted by the $b_{\max }$ values increasing rainbow angles. The trend appears for transitions to $J=3$ too, but not for transitions to $J=4$. This could mean that the DCS is a more complicated function of all the quantum numbers, both in the initial and final level. In addition, such propensities may not be observed in the measured DCSs because the latter are thermal averages of processes starting from two initial levels. [25] 

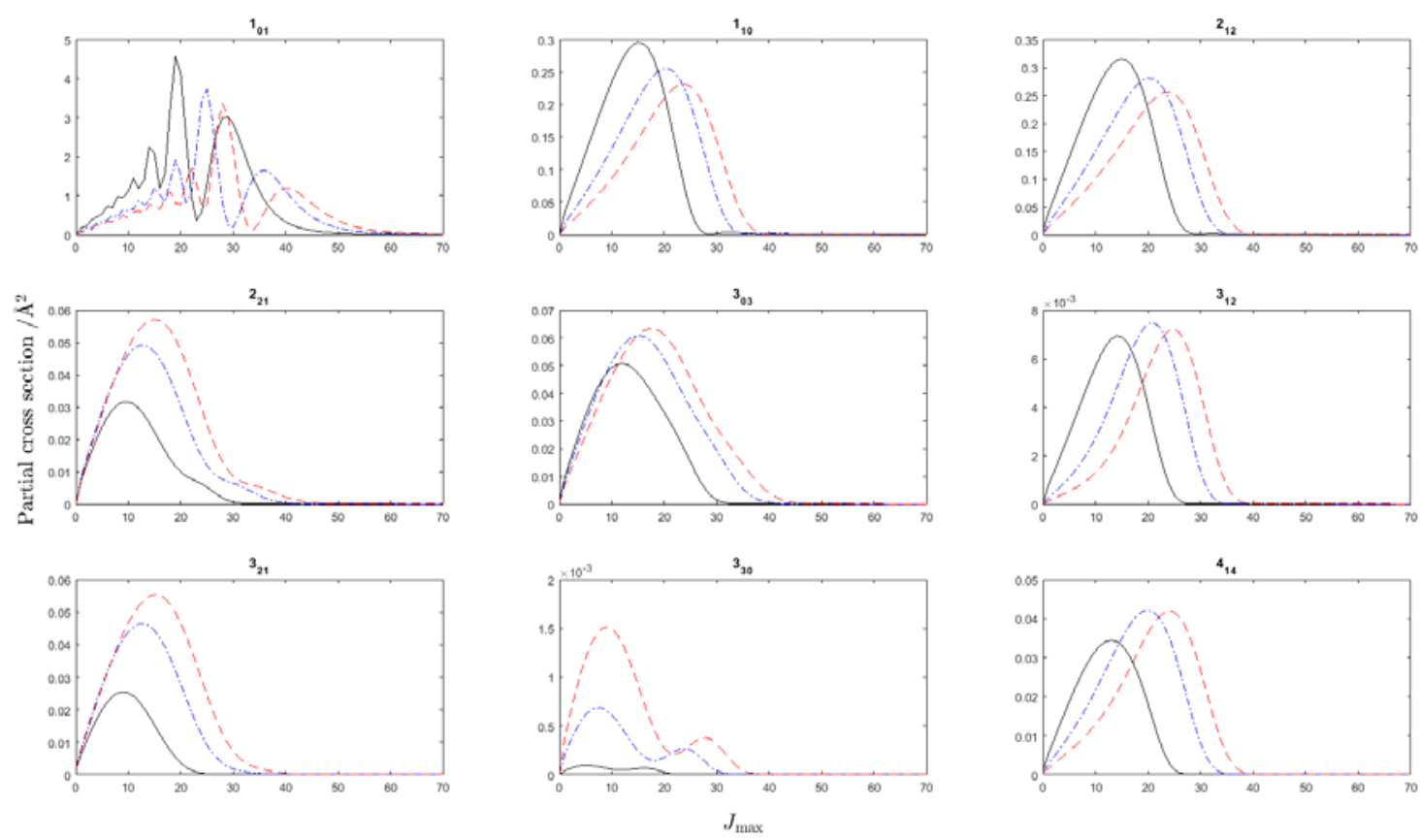

Figure 10: Close-coupling quantum mechanical partial cross sections (PCS) for the $\mathrm{He}+\mathrm{H}_{2} \mathrm{O}\left(1_{01}\right)$ at $E_{\text {col }}=$ 381.34 (solid line), 601.90 (dash-dotted line) and 763.17 (dashed line) $\mathrm{cm}^{-1}$. The final $\mathrm{H}_{2} \mathrm{O}$ rotational levels are shown in the title of each graph, and the DCSs are presented in increasing rotational energy of the final level. 


\section{Conclusions}

At high collision energies, a common assumption is that the atoms of two colliding particles approach closely, and scattering is controlled by the repulsive part of the potential. However, we see a reverse case in our experiment at high collision energies. The very forward nature of $\mathrm{H}_{2} \mathrm{O}+\mathrm{He}$ scattering at high collision energies can be a signature of scattering with large impact parameters. This is verified in the close-coupling quantum mechanical partial cross sections shown in Fig. 10. The shape of the partial cross sections at higher collision energies is compatible with a forward scattered collision that samples a less repulsive part of the potential energy surface.

It seems that at higher collision energies, the head-on collisions, which usually give backward scattered products, lead into higher final rotational levels and, therefore, decrease the backward scattered products for low rotational excitations measured here. Therefore, at high collision energies, the ICS for low rotational excitation of $\mathrm{H}_{2} \mathrm{O}$ decreases, and shows more forward scattering in comparison to higher excitation. Similar conclusions have been derived previously for the DCS in the $\mathrm{H}+\mathrm{D}_{2} \rightarrow \mathrm{HD}\left(v^{\prime}=1, j^{\prime}=2,6,10\right)+\mathrm{D}$ reaction, where an increase in collision energy changes only weakly the scattering angle distribution of a specific $J$ level mainly indirectly by its effect on the rotational distribution.[30]

These observations show that the correlation between the amount of rotational energy transfer and the collision energy of the system can be examined using DCS measurements. The calculation of quantum mechanical PCS helped to understand the dynamics that govern the $\mathrm{H}_{2} \mathrm{O}+\mathrm{He}$ collisions. Further investigations on similar systems, such as $\mathrm{H}_{2} \mathrm{O}+\mathrm{Ar} / \mathrm{Xe}$ and extending the studies to other final rotational levels will provide a complete picture of the collisions. As Kolb et al. suggested impact only on the $\mathrm{H}$ atoms of $\mathrm{H}_{2} \mathrm{O}$ can cause rotation around the $b$ axis. [31] Future quasi-classical trajectories (QCT) calculations may reveal more details about the mechanism of the rotational excitation. 


\section{Acknowledgements}

This research utilized Queen Mary's MidPlus computational facilities, supported by QMUL Research-IT and funded by EPSRC grant EP/K000128/1. We acknowledge Dr B. Yang (The University of Georgia) for sending the ICS values used in Table 2. S. M. would like to thank Profs. M. H. Alexander and P. J. Dagdigian for their continuous support without which the theoretical part of this work would not have been completed. L. M. was supported by a grant from the BBSRC to R. W. Janes (BB/J019194/1). Computations in Grenoble were performed at the CIMENT infrastructure (https://ciment.ujfgrenoble.fr).

\section{References}

[1] B. Nisini, Astronomy - Water's role in making stars, Science 290 (2000) 1513-1514.

[2] A.C. Cheung, D.M. Rank, C.H. Townes, D.D. Thornton, W.J. Welch, Detection of Water in Interstellar Regions by its Microwave Radiation, Nature 221 (1969) 626-628.

[3] E.F. van Dishoeck, E. Herbst, D.A. Neufeld, Interstellar Water Chemistry: From Laboratory to Observations, Chem. Rev. 113 (2013) 9043-9085.

[4] S. Green, Collisional Excitation of Interstellar Molecules: Water, Astrophys J Suppl S 42 (1980) 103-141.

[5] R.W. Bickes Jr, G. Duquette, C.J.N. van den Meijdenberg, A.M. Rulis, G. Scoles, K.M. Smith, Molecular beam scattering experiments with polar molecules: measurement of differential collision cross sections for $\mathrm{H}_{2} \mathrm{O}+\mathrm{H}_{2}, \mathrm{He}, \mathrm{Ne}, \mathrm{Ar}, \mathrm{H}_{2} \mathrm{O}$ and $\mathrm{NH}_{3}+\mathrm{H}_{2}, \mathrm{He}, \mathrm{NH}_{3}$, J. Phys. B: Atom. Molec. Phys. 8 (1975) 3034-3043.

[6] M. Keil, J.T. Slankas, A. Kuppermann, Scattering of thermal He beams by crossed atomic and molecular beams. II. The He-Ar van der Waals potential, J. Chem. Phys. 70 (1979) 482-503.

[7] J. Brudermann, C. Steinbach, U. Buck, K. Patkowski, R. Moszynski, Elastic and rotationally inelastic differential cross sections for $\mathrm{He}+\mathrm{H}_{2} \mathrm{O}$ collisions, J. Chem. Phys. 117 (2002) 11166-11174.

[8] D. Cappelletti, V. Aquilanti, E. Cornicchi, M.M. Teixidor, F. Pirani, Molecular-beam study of the water-helium system: Features of the isotropic component of the intermolecular interaction and a critical test for the available potential-energy surfaces, J. Chem. Phys. 123 (2005) 024302.

[9] M. Ivanov, M.L. Dubernet, Rotational quenching of $\mathrm{H}_{2} \mathrm{O}$ by He: Mixed quantum/classical theory and comparison with quantum results, J. Chem. Phys. 140 (2014) 134301.

[10] A. Semenov, M.L. Dubernet, D. Babikov, Mixed quantum/classical theory for inelastic scattering of asymmetric-top-rotor + atom in the body-fixed reference frame and application to the $\mathrm{H}_{2} \mathrm{O}+\mathrm{He}$ system, J. Chem. Phys. 141 (2014) 114304.

[11] W.B. Chapman, A. Kulcke, B.W. Blackmon, D.J. Nesbitt, Rotationally inelastic scattering of jet cooled $\mathrm{H}_{2} \mathrm{O}$ with Ar: State-to-state cross sections and rotational alignment effects, J. Chem. Phys. 110 (1999) 8543-8554.

[12] C.-H. Yang, G. Sarma, J.J. ter Meulen, D.H. Parker, U. Buck, L. Wiesenfeld, Imaging the Inelastic Scattering of Water with Helium. Comparison of Experiment and Theory, J. Phys. Chem. A 114 (2010) 9886-9892.

[13] C.-H. Yang, G. Sarma, J.J. ter Meulen, D.H. Parker, G.C. McBane, L. Wiesenfeld, A. Faure, Y. Scribano, N. Feautrier, Communication: Mapping water collisions for interstellar space conditions, J. Chem. Phys. 133 (2010) 131103.

[14] K. Patkowski, T. Korona, R. Moszynski, B. Jeziorski, K. Szalewicz, Ab initio potential energy surface and second virial coefficient for He-H2O complex, J. Mol. Struct. (Theochem) 591 (2002) 231.

[15] M.P. Hodges, R.J. Wheatley, A.H. Harvey, Intermolecular potential and second virial coefficient of the water-helium complex, J. Chem. Phys. 116 (2002) 1397-1405.

[16] G. Calderoni, F. Cargnoni, M. Raimondi, An ab initio investigation of the He-H2O complex, Chem. Phys. 
Lett. 370 (2003) 233-239.

[17] B. Yang, P.C. Stancil, Close-coupling study of rotational energy transfer and differential scattering in $\mathrm{H} 2 \mathrm{O}$ collisions with He atoms, J. Chem. Phys. 126 (2007) 154306.

[18] HIBRIDON is a package of programs for the time-independent quantum treatment of inelastic collisions and photodissociation written by M. H. Alexander, D. Manolopoulos, H.-J. Werner, and B. Follmeg, with contributions by P. F. Vohralik, D. Lemoine, G. Corey, R. Gordon, B. Johnson, T. Orlikowski, A. Berning, A. Degli-Esposti, C. Rist, P. Dagdigian, B. Pouilly, G. van der Sanden, M. Yang F. de Weerd, S. Gregurick, and J. Kłos.

[19] D.E. Manolopoulos, An improved log derivative method for inelastic scattering, J. Chem. Phys. 85 (1986) 6425-6429.

[20] M.H. Alexander, D.E. Manolopoulos, A stable linear reference potential algorithm for solution of the quantum close-coupled equations in molecular scattering theory, J. Chem. Phys. 86 (1987) 2044-2050.

[21] P.J. Dagdigian, M. Alexander, Depolarization in H2O-He collisions, Mol. Phys. 108 (2010) 1159-1169.

[22] J. Tennyson, P.F. Bernath, L.R. Brown, A. Campargue, A.G. Császár, L. Daumont, R.R. Gamache, J.T. Hodges, O.V. Naumenko, O.L. Polyansky, L.S. Rothman, A.C. Vandaele, N.F. Zobov, A.R. Al Derzi, C. Fábri, A.Z. Fazliev, T. Furtenbacher, I.E. Gordon, L. Lodi, I.I. Mizus, IUPAC critical evaluation of the rotational-vibrational spectra of water vapor, Part III: Energy levels and transition wavenumbers for $\mathrm{H}_{2}{ }^{16} \mathrm{O}$, J. Quant. Spectrosc. Radiat. Transfer 117 (2013) 29-58.

[23] B. Yang, M. Nagao, W. Satomi, M. Kimura, P.C. Stancil, Rotational quenching of rotationally excited H2O in collisions with He, Astrophys J. 765 (2013) 77.

[24] J. M. Hutson and S. Green, MOLSCAT computer code, version 14 (1994), distributed by Collaborative Computational Project No. 6 of the Engineering and Physical Sciences Research Council (UK).

[25] G. Sarma, C.-H. Yang, A.K. Saha, D.H. Parker, L. Wiesenfeld, Rotational excitation of $\mathrm{HDO}$ and $\mathrm{D}_{2} \mathrm{O}$ by $\mathrm{H}_{2}$ : Experimental and theoretical differential cross-sections, J. Chem. Phys. 138 (2013) 024314.

[26] S. Bosanac, Two-dimensional model of rotationally inelastic collisions, Phys. Rev. A 22 (1980) 2617-2622.

[27] S. Bosanac, U. Buck, Rotational rainbow scattering from an off-center rigid shell model, Chem. Phys. Lett. 81 (1981) 315-319.

[28] J.N. Murrell, S.D. Bosanac, Introduction to the Theory of Atomic and Molecular Collisions, John Wiley \& Sons, Chichester, 1989.

[29] R. Schinke, H.J. Korsch, D. Poppe, Rainbows in rotationally inelastic scattering: A comparative study of different model potential surfaces and dynamical approximations, J. Chem. Phys. 77 (1982) 6005-6020.

[30] K. Koszinowski, N.T. Goldberg, J. Zhang, R.N. Zare, F. Bouakline, S.C. Althorpe, Differential cross section for the $H+D_{2} \rightarrow H D\left(v^{\prime}=1, j^{\prime}=2,6,10\right)+D$ reaction as a function of collision energy, J. Chem. Phys. 127 (2007) 124315.

[31] C.E. Kolb, H.R. Baum, K.S. Tait, Classical calculations of $\mathrm{H}_{2} \mathrm{O}$ rotational excitation in energetic atom-molecule collisions, J. Chem. Phys. 57 (1972) 3409. 